\title{
SEMA6A/RhoA/YAP Axis Mediates Tumor-Stroma Interactions and Prevents Response to Dual BRAF/MEK Inhibition in BRAF-Mutant Melanoma
}

Rossella Loria

Regina Elena Institute: Istituto Regina Elena

Valentina Laquintana

Regina Elena Institute: Istituto Regina Elena

Stefano Scalera

Regina Elena Institute: Istituto Regina Elena

Rocco Fraioli

Regina Elena Institute: Istituto Regina Elena

Valentina Caprara

Regina Elena Institute: Istituto Regina Elena

Italia Falcone

Regina Elena Institute: Istituto Regina Elena

Chiara Bazzichetto

Regina Elena Institute: Istituto Regina Elena

Marta Di Martile

Regina Elena Institute: Istituto Regina Elena

Laura Rosanò

National Research Council: Consiglio Nazionale delle Ricerche

Donatella Del Bufalo

Regina Elena Institute: Istituto Regina Elena

Gianluca Bossi

Regina Elena Institute: Istituto Regina Elena Isabella Sperduti

Regina Elena Institute: Istituto Regina Elena Irene Terrenato

Regina Elena Institute: Istituto Regina Elena

\section{Paolo Visca}

Regina Elena Institute: Istituto Regina Elena

\section{Silvia Soddu}

Regina Elena Institute: Istituto Regina Elena

Michele Milella 
University of Verona: Universita degli Studi di Verona

\section{Gennaro Ciliberto}

Regina Elena Institute: Istituto Regina Elena

\section{Rita Falcioni}

Regina Elena Institute: Istituto Regina Elena

\section{Virginia Ferraresi}

Regina Elena Institute: Istituto Regina Elena

\section{Giulia Bon ( $\nabla$ giulia.bon@ifo.gov.it )}

Istituto Regina Elena https://orcid.org/0000-0002-5409-5864

\section{Research Article}

Keywords: semaphorin SEMA6A, melanoma, dual BRAF/MEK inhibition, actin cytoskeleton remodeling, YAP, tumor microenvironment

Posted Date: November 11th, 2021

DOI: https://doi.org/10.21203/rs.3.rs-1053824/v1

License: (1) (1) This work is licensed under a Creative Commons Attribution 4.0 International License. Read Full License

Version of Record: A version of this preprint was published at Journal of Experimental \&amp; Clinical Cancer Research on April 19th, 2022. See the published version at https://doi.org/10.1186/s13046-02202354-w. 


\section{Abstract}

Background: Despite the promise of dual BRAF/MEK inhibition as a therapy for BRAF-mutant (BRAF-mut) melanoma, heterogeneous responses have been observed in patients, thus predictors of benefit from therapy are needed. We have previously identified semaphorin 6A (SEMA6A) as a BRAF-mut-associated protein involved in actin cytoskeleton remodeling. The purpose of the present study is to dissect the role of SEMA6A in the biology of BRAF-mut melanoma, and to explore its predictive potential towards dual BRAF/MEK inhibition.

Methods: SEMA6A expression was assessed by immunohistochemistry in melanoma cohort RECl1 $(\mathrm{N}=112)$ and its prognostic potential was investigated in BRAF-mut melanoma patients from DFCl and TCGA datasets $(\mathrm{N}=258)$. The molecular mechanisms regulated by SEMA6A to sustain tumor aggressiveness and targeted therapy resistance were investigated in vitro by using BRAF-mut and BRAFwt melanoma cell lines, an inducible SEMA6A silencing cell model and a microenvironment-mimicking fibroblasts-coculturing model. Finally, SEMA6A prediction of benefit from dual BRAF/MEK inhibition was investigated in melanoma cohort RECI2 ( $\mathrm{N}=14)$.

Results: Our results indicate higher protein expression of SEMA6A in BRAF-mut compared with BRAF-wt melanoma patients and show that SEMA6A is a prognostic indicator in BRAF-mut melanoma from TCGA and DFCl patients cohorts. In BRAF-mut melanoma cells, SEMA6A coordinates actin cytoskeleton remodeling by the RhoA-dependent activation of YAP and dual BRAF/MEK inhibition by dabrafenib+trametinib induces SEMA6A/RhoA/YAP axis. In microenvironment-mimicking co-culture condition, fibroblasts confer to melanoma cells a proliferative stimulus and protect them from targeted therapies, whereas SEMA6A depletion rescues the efficacy of dual BRAF/MEK inhibition. Finally, in BRAFmut melanoma patients treated with dabrafenib+trametinib, high SEMA6A predicts shorter recurrencefree interval.

Conclusions: Overall, our results indicate that SEMA6A contributes to microenvironment-coordinated evasion of melanoma cells from dual BRAF/MEK inhibition and it might be a good candidate predictor of short-term benefit from dual BRAF/MEK inhibition.

\section{Background}

Melanoma is characterized by the higher frequency of genetic (1) and epigenetic (2-4) deregulations among all tumor type, with the majority of mutations affecting the mitogen-activated protein kinase (MAPK) pathway (5). BRAF mutation is the most common MAPK pathway aberration, occurring in 40$60 \%$ of melanoma cases. The introduction of BRAF-targeted therapies (6) and, more recently, of combination therapy with BRAF and MEK inhibitors, such as dabrafenib+trametinib (7-9) has resulted in dramatic improvements in terms of overall survival (OS) and progression-free survival (PFS) in patients affected by BRAF-mutant (BRAF-mut) advanced melanoma. However, some patients benefit from dual 
BRAF/MEK inhibition much more than others (9-11), indicating that the identification of novel predictive biomarkers is needed to optimize individualized treatment strategies.

By an unbiased screening of different single cell clones derived from the same melanoma patient and mutually carrying BRAF ${ }^{\mathrm{V} 600 \mathrm{E}}$ or NRAS ${ }^{\mathrm{Q} 61 \mathrm{R}}$ mutations, we previously identified semaphorin SEMA6A as a protein whose expression is associated with $\mathrm{BRAF}^{\mathrm{V} 600 \mathrm{E}}$ mutation. In addition, we observed that SEMA6A regulates actin cytoskeleton remodeling, thereby sustaining proliferation and survival of BRAF-mut melanoma cells (12). Later on, SEMA6A deregulation in BRAF ${ }^{\mathrm{V} 600 \mathrm{E}}$ as compared with BRAF wt melanoma patients has been reported by others (13).

Semaphorins belong to a family including more than 20 members; some of them have been shown to modulate angiogenesis, invasiveness, and metastatization by regulating monomeric GTPases, cellsubstrate adhesion, and cytoskeletal dynamics (14-16). More recently, the involvement of semaphorins in the complex signal exchange between tumor and its microenvironment is emerged and supported by a large body of evidence (17-25). Of relevance, tumor microenvironment plays an important role in protecting cancer cells from the anti-tumor activity of BRAF and MEK inhibitors through paradoxic upregulation of signaling pathways and survival factors (26-29). A very first response of cancer cells to stimuli from tumor microenvironment is represented by actin cytoskeleton remodeling to promote survival, migration, and metastatization $(30,31)$. A critical role in this process is played by the Rho family of small GTPases that, through the activation of downstream targets including YAP, cohordinates stress fibers and actin bundles formation (32).

YAP is a coactivator of transcription factors and a Hippo suppressor pathway transducer with tumorigenic potential in mouse model (33) and pro-invasive properties in melanoma (34). Nuclear accumulation of YAP has been described in a variety of cancers (35-39) resulting in activation of target genes mainly involved in cell proliferation. Remarkably, YAP activation has been reported to be associated with stemness (40), conversion of normal fibroblasts to cancer-associated fibroblasts (CAFs) (41) and resistance to BRAF and/or MEK inhibitors $(42,43)$. Specifically, YAP confers resistance to BRAF inhibitors by inducing actin cytoskeleton remodeling in melanoma (44) and other tumors (45), and cytoskeletal tension itself has been shown to affect chemotherapeutic drug sensitivity of cancer cells (46).

In the present study, we observed that SEMA6A expression is higher in BRAF-mut than in BRAF-wt melanoma patients and has a prognostic significance in BRAF-mut patients from TCGA and DFCl large cohorts. Mechanistically, we show that in BRAF-mut melanoma cells, SEMA6A remodels actin cytoskeleton by activating the RhoA-YAP axis. Furthermore, the activity of SEMA6A/RhoA/YAP axis is induced by dual BRAF/MEK inhibition by dabrafenib+trametinib and, in an environmental-mimicking condition of melanoma cells and fibroblasts co-culture, is associated with reduced targeted therapy efficacy. These results were confirmed in BRAF-mut melanoma patients treated with dabrafenib+trametinib, where high SEMA6A expression predicts shorter progression-free survival upon therapy. Overall these data support the involvement of SEMA6A in fibroblasts-induced protection of 
melanoma cells from the anti-tumor activity of dual BRAF/MEK inhibition and indicate that SEMA6A might be a good candidate predictor of short-term benefit from dual BRAF/MEK blockade.

\section{Methods}

\section{Cell lines, co-culture, and treatments.}

The Human Fibroblast BJ were obtained from the American Type Culture Collection (ATCC) and maintained in DMEM medium supplemented with $10 \%$ FBS and 1\% penicillin/streptomycin (Gibco, Life Technologies, Milan, Italy). BRAF-wt/NRAS-wt ME1007 and BRAF-wt/NRAS-mut ME4405, derived from lymph node metastases, and BRAF-wt/NRAS-mut 2/17 and BRAF ${ }^{V 600 E} /$ NRAS-wt 2/59, derived from subcutaneous metastasis, were isolated from surgical specimens of melanoma patients, not previously subjected to chemotherapy and admitted to Fondazione IRCCS Istituto Nazionale dei Tumori, Milan (Sensi M et al, 2006; Daniotti M et al, 2004). M14 and C32 cell lines were obtained from the American Type Culture Collection (ATCC).

To obtain an inducible SEMA6A depletion, 2/59 cells were transduced using shERWOOD UltramiR lentiviral inducible shRNA ( $\mathrm{pZIP-TRE3G)} \mathrm{according} \mathrm{to} \mathrm{the} \mathrm{manufacturer's} \mathrm{guidelines} \mathrm{(Transomic}$ Technologies, Huntsville, AL USA). Stable cells were generated by antibiotic selection with $1 \mu \mathrm{g} / \mathrm{mL}$ puromycin for 14 days. We obtained a control cell line by using a non-specific shRNA (2/59 shCtrl) and two cell lines inducible for SEMA6A depletion by using two specific shRNAs (2/59 shSEMA6A A3 and $\mathrm{H} 2$ ). To induce shRNAs and GFP reporter gene expression, cells were treated with doxycycline at 0,5 $\mu \mathrm{g} / \mathrm{mL}$. All melanoma cell lines were maintained in RPMI medium supplemented with $10 \%$ FBS and $1 \%$ penicillin/streptomycin (Gibco, Life technologies, Milan, Italy). All cell lines were periodically tested for mycoplasma contamination.

For co-cultures, BJ were plated and three days after 2/59 shCtrl, A3 and $\mathrm{H} 2$ were added to BJ monolayer cultures without discard the culture medium.

For treatments in vitro, trametinib and dabrafenib (Selleckchem, Houston, TX, USA) were used at $5 \mathrm{nM}$ and $0,1 \mathrm{mM}$ concentrations respectively. The drugs were stored as stock solutions at $-20^{\circ} \mathrm{C}$ and diluted just before use.

\section{Antibodies}

Anti-Sema6A (ab72369) was from Abcam (Cambridge, UK), anti-Lamin A (\#86846), anti-Akt (\#9272), anti-phospho-ser473-Akt (\#9271), anti-Erk1/2 (\#9102), anti-phospho-Thr202/Tyr204-Erk 1/2 (\#9101), anti-YAP (\#12395), anti-phospho-ser127-YAP (\#13008), anti-p65 (\#8242), anti-phospho-ser536-p65 (\#3033), anti-Tubulin (\#2125) were from Cell Signaling (Danvers, MA USA), anti-Hsp-70 (ab-83392) and anti-GAPDH (ab-81594) were from Immunological Sciences (Rome, Italy). HRP-conjugated secondary antibodies were from Bio-Rad (Hercules, CA USA). 


\section{Western Blotting}

All cell lines, before and after treatment, were lysed using RIPA buffer, analyzed by SDS-PAGE and probed (WB) with antibodies of interest and secondary HRP-conjugated antibodies. Signals were detected by Amersham ECL Prime Western Blotting Detection Reagent (GE Healthcare Life Sciences, Chicago, IL USA) and by LuminataTM Classico Western HRP substrate (Millipore, Burlington, MA USA). Same amount of total protein from three independent experiments were pooled and analyzed. Each experiment was repeated at least three times.

\section{Nucleic and cytoplasmic fractionation}

Nucleic and cytoplasmic fractions, derived from 2/59 shCtrl and shSEMA6A A3 and H2 cells 72 hours post-induction, untreated or treated with RhoA activator I, were obtained using NE-PER Nuclear and Cytoplasmic Extraction Reagents (\#78835; Thermo Scientific, Rockford IL USA) according to the manufacturer's guidelines. Each experiment was repeated at least three times.

\section{RhoA Activation Assay and Immunofluorescence analysis}

2/59 shCtrl and shSEMA6A A3 and H2 cells were plated on $100 \mathrm{~mm}$ dishes or poly-l lysine coated slides 24 hours post-induction. After 48 hours cells were treated with $1 \mathrm{U} / \mathrm{mL}$ RhoA activator $\mathrm{I}$, according to the manufacturer's guidelines (\#CN01; Cytoskeleton, Denver, CO USA). The levels of RhoA activation in untreated or treated cells were analyzed by RhoA activation assay (\#BK036; Cytoskeleton, Denver CO USA) performed according to the manufacturer's guidelines.

For immunofluorescence experiments, cells untreated or treated with RhoA activator I as described above were stained with anti-YAP (sc-376830; Santa Cruz, Dallas TX, USA) or Alexa Fluor 555 Phalloidin (\#8953; Cell Signaling, Danvers, USA) and counterstained with Hoechst to highlight nuclei (SIGMAAldrich, St. Luis, MO USA). 2/59 and ME4405 cells plated on poly-l lysine coated slides were treated with trametinib, dabrafenib and their combination, and stained as above. The quantification of YAP localization was performed by inspecting at least five fields/slide using a 60X magnification in three independent experiments.

Microscope OLYMPUS BX53 was used to evaluate fluorescence. Scale bars $10 \mu \mathrm{m}$. Each assay was carried out in triplicate and repeated at least three times.

\section{Crystal Violet Assay}

Following doxycycline induction for 24 hours, 2/59shCtrl and shSEMA6A A3 and $\mathrm{H} 2$ cells plated on 24well plates were treated with trametinib, dabrafenib and/or their combination at specified concentrations. 72 hours later the cells were fixed with $4 \%$ formaldehyde for 15 minutes and stained with $0.1 \%$ crystal violet solution in $10 \%$ ethanol for 40 minutes. At the end of incubation the medium was removed, cells were allowed to dry at room temperature and dissolved in isopropanol, and optical density was measured 
at $570 \mathrm{~nm}$ using an ELISA plate reader. Each assay was carried out in quadruplicate and repeated at least three times.

\section{Cell sorting and IncuCyte® analyses}

BJ were plated on 12-well plates or $60 \mathrm{~mm}$ dishes and three days later shCtrl, A3 and A H2 2/59, following a 72 hours-induction, were added to BJ monolayer cultures without discard the culture medium. The day after cells were treated with trametinib, dabrafenib or their combination.

After 48 hours melanoma cells seeded on $60 \mathrm{~mm}$ dishes were separated from the fibroblasts by BD FACSMelody Cell Sorter, gating on the GFP signal and collecting only clearly GFP positive melanoma cells. The purity of the FACS-separated melanoma fractions (i.e. no contamination with fibroblasts) is guaranteed since the gating was stringent. Total cell lysate extracted from sorted melanoma cells were analyzed by western blot.

Co-culture performed on 12-well plates were incubated in IncuCyte ${ }^{\circledR}$ and monitored non-invasively by a planned acquisition of images every 12 hours for 156-180 hours. Images were analyzed by software supplied by Sartorius. Each experiment was carried out in triplicate and repeated at least three times.

\section{Patient cohorts}

For SEMA6A protein expression two patient cohorts were used; the RECl1 cohort, including 59 BRAF ${ }^{V 600 E}$ and 53 BRAF-wt melanoma patients treated at the Regina Elena National Cancer Institute, was used to compare SEMA6A expression in BRAF-mut versus BRAF-wt tumors. Bioptic specimens were collected from the metastatic lesions of involved patients. The RECl2 cohort was used to assess the predictive role of SEMA6A expression in 14 BRAF-mut melanoma patients admitted to dabrafenib+trametinib at Regina Elena National Cancer Institute. Based on recurrence-free interval, RECI2 patients were categorized as short-term and long-term responders; a cut-off value of 12 months was established. Information on clinical and histopathological features, anti-tumoral therapies and related outcomes were retrieved from patients' medical records by specifically trained research assistants; written informed consent from all patients was obtained in accordance with the Declaration of Helsinki. TCGA (DOI:

10.1016/j.cell.2018.03.022) and DFCI (DOI: 10.1038/s41591-019-0654-5) melanoma cohorts were used to investigate the prognostic role of SEMA6A in BRAF-mut melanoma. Melanoma cases were referred to as "early" when stage was 0 , I, and II, and "advanced" when stage was III and IV. Genomic and clinical data from the two cohorts were downloaded from cBioportal. Only patients with BRAF mutation were considered for the analyses. RNA-seq data were obtained from Firebrowse (http://firebrowse.org/). SEMA6A expression from RNA-seq data was quantified through TPM (Transcripts per Million) normalization. Patients were classified as SEMA6A High and Low expression by calculating the relative TPM value for each sample in the patient cohort $(\mathrm{N}=20)$.

\section{Immunohistochemistry}


Formalin-fixed paraffin-embedded sections were analyzed as described [41]. Antigen retrieval was performed at $96^{\circ} \mathrm{C}(10 \mathrm{mM} / \mathrm{L}$ citrate buffer, $\mathrm{pH} 6)$ for 20 minutes. Sections from specimens of RECl1 and RECl2 cohorts were incubated with the primary antibody anti-SEMA6A 1:50 (HPA031265; SIGMA-Aldrich, St. Luis, MO USA) for 30 minutes at room temperature. Immunoreactions were revealed by Bond Polymer Refine Detection Kit according to manufacturer's procedure (Leica Biosystems) in an automated autostainer Bond III Leica Biosystems. Diaminobenzidine was used as chromogenic substrate. Microscope Nikon ECLIPSE 55i with digital camera HESP Technology was used. Scale bars $30 \mu \mathrm{m}$. The study was reviewed and approved by the ethical committee of Regina Elena National Cancer Institute, and informed consent was obtained from all patients.For IHC, the secondary antibody was used as internal control.

\section{Statistical analyses}

Data were reported as the mean of the three replicates \pm standard deviation (SD). Each experiment was performed independently. To compare continuous variables two-tailed Student's t-tests with BenjaminiHochberg correction was performed while Pearson's Chi-square test was used to compare categorical variables. One-way ANOVA with Tukey HSD test was used for multiple sample comparison. Survival analysis was conducted through the use of Kaplan-Meier method and the Log-rank test was used to individuate potential differences between subgroups. P-value $<0.05$ was considered significant.

Statistical analyses were performed with SPSS software (SPSS version 21.0, SPSS Inc., Chicago, IL) and in-house scripting in $\mathrm{R}$ environment.

\section{Results}

\section{SEMA6A is highly expressed and has prognostic relevance in BRAF-mut melanoma patients.}

We have previously shown preferential expression of SEMA6A in BRAF-mut cell lines and melanoma lymph-node metastases compared with BRAF-wt ones (12). We first verified the association of SEMA6A protein expression with BRAF mutation in the RECl1 cohort, which includes 112 metastatic advanced melanoma patients surgically treated at the Regina Elena National Cancer Institute. SEMA6A protein expression was measured by immunohistochemistry (IHC) and assigned an intensity score (IS) value ranging from 0 to 3 . We then dichotomized SEMA6A into two categories: "low' if IS was 0 or 1 and "high" if score was 2 or 3 . Table 1 shows the main patient- and tumor-related characteristics. Briefly, 59 patients $(52,7 \%)$ were affected by BRAF-mut disease and $53(47,3 \%)$ were BRAF-wt. SEMA6A was high in 36 $(32,1 \%)$ and low in $76(67,9 \%)$ patients. As shown in Figure 1A and in representative IHC panels of Figure 1B, we found a statistically significant association between high SEMA6A and BRAF mutation (Pearson's Chi-square test $p<0,0001$ ) (Figure $1 A$ ). Notably, $100 \%$ of samples with SEMA6A IS 3 were BRAF-mut. In addition, we performed SEMA6A IHC in subsequent metastatic lesions at diagnosis and at progression in three patients. As shown in Table 2, SEMA6A levels progressively increased, suggesting that SEMA6A may have prognostic relevance in BRAF-mut melanoma. To verify this hypothesis, we analyzed mRNA data from TCGA and DFCI large datasets; advanced and early melanoma patients were analyzed 
separately. When focusing on patients with advanced melanoma $(n=149)$, patients with high SEMA6A expression had reduced PFS and OS than those with low SEMA6A (PFS: 6.76 months vs. 19.50 months; Log-Rank p=0.013; OS: 31.6 months vs. 69.1 months; Log-Rank 0.0008) (Figures 1C and 1D). In patients with early melanoma $(n=109)$, high SEMA6A was significantly associated with reduced relapse-free survival (RFS) (40.5 months vs. 55.5 months; Log-Rank $p=0.031$ ) (Figure 1E). Overall these data support the involvement of SEMA6A in the biology of BRAF-mut melanoma and indicate that SEMA6A is a prognostic indicator in this subset of patients.

\section{SEMA6A regulates actin cytoskeleton remodeling by activating the RhoA/YAP axis in BRAF-mut melanoma cells.}

We have previously identified SEMA6A as a BRAF-mut-associated protein that might contribute to actin cytoskeleton remodeling (12). Our previous findings indicate that the depletion of SEMA6A by siRNA has dramatic effect in terms of cell death; thus, to unravel the molecular mechanism by which SEMA6A regulates actin stress fibers dynamics, we aimed to exploit a cell model that would allow us to modulate SEMA6A expression and avoid massive cell death. For this purpose, the BRAF-mut melanoma cell line 2/59 was engineered for inducible silencing of SEMA6A expression and induction of GFP reporter gene expression. We obtained three polyclonal cell populations: one shCtrl and two shSEMA6A ( $\mathrm{A} 3$ and $\mathrm{H} 2)$. Following induction, the expression of SEMA6A was efficiently downregulated in A3 and $\mathrm{H} 2$ compared with shCtrl cells (Figure $2 \mathrm{~A}$ ), resulting in $20-30 \%$ reduction in the number of viable melanoma cells (Figure 2B). In agreement with our previous findings, the depletion of SEMA6A in 2/59 cell model induced actin cytoskeleton remodeling and loss of actin stress fibers (Figure 2C).

Next, we investigated the involvement of Rho small GTPases and downstream YAP in SEMA6A-induced actin cytoskeletal remodeling. First, we measured the protein levels of Rho GTPs in 2/59 cells and found the expression of RhoA but not of RhoC small GTPase (data not shown); thus we analyzed the level of active RhoA-GTP upon induction of SEMA6A silencing. As shown in Figure 2D, SEMA6A depletion caused a reduction of RhoA activity compared with control cells. The densitometric analysis of pooled immunoblot data from both $\mathrm{H} 2$ and $\mathrm{A} 3$ revealed a statistically significant reduction of RhoA activity upon SEMA6A depletion (Figure 2E). Next, we found higher levels of YAP phosphorylation following SEMA6Adepletion (Figure 2A). Moreover, Wester Blot analysis of cytoplasmic and nuclear fractions of control and SEMA6A-depleted cells revealed increased phosphorylation levels of YAP and its cytoplasmic retention in $\mathrm{A} 3$ and $\mathrm{H} 2$ compared with shCtrl cells (Figure 2F, upper panels). The densitometric analysis of pooled data from both $\mathrm{H} 2$ and $\mathrm{A} 3$ shows increase of YAP phosphorylation in the cytoplasmic fraction of SEMA6A-depleted cells (Figure 2G). YAP cytoplasmic accumulation following SEMA6A depletion was confirmed by immunofluorescence (Fig. $2 \mathrm{H}$, upper panels) and was associated with loss of stress fibers (Figure 2I, upper panels). We then investigated whether induction of RhoA activity by a specific activator can rescue RhoA-YAP signaling in SEMA6A-depleted cells. To this aim, shCtrl, A3 and $\mathrm{H} 2$ cells were treated with RhoA activator and analyzed as above. We observed a significant induction of RhoA activity (Figure 2D and 2E) and the reduction of YAP cytoplasmic localization (Figure 2F and Figure 2H, upper panels) in shCtrl cells compared with their untreated counterpart. By contrast, treatment of SEMA6A- 
depleted cells with the RhoA activator did not rescue the level of active RhoA-GTP (Figure 2D and 2E), YAP cytoplasmic localization (Figure 2F, lower panels, and Figure $2 \mathrm{H}$, lower panels), and actin cytoskeleton organization (Figure 2I, lower panels), indicating that SEMA6A is required for downstream activation of RhoA/YAP axis. These data show that SEMA6A regulates actin cytoskeleton remodeling by sustaining the activity of RhoA that in turn induces YAP nuclear localization.

\section{Dual BRAF/MEK inhibition induces SEMA6A/RhoA/YAP axis.}

YAP-induced actin cytoskeleton remodeling has been recently reported as a mechanism of resistance to BRAF inhibitors $(44,45)$. Thus, we investigated the activity of SEMA6A/RhoA/YAP axis in response to targeted therapy by treating BRAF-mut cell lines 2/59, M14 and C32 with the BRAF inhibitor dabrafenib, the MEK inhibitor trametinib, and the combination of the two drugs. All treatments inhibited the phosphorylation of ERK 1/2 (p-ERK) as expected and induced a marked increase of SEMA6A expression in all the three BRAF-mut cell lines (Figure 3A, left panels). The increase of SEMA6A expression was accompanied by a mild but reproducible induction of RhoA activity (Figure $3 \mathrm{~B}$ and $3 \mathrm{C}$ ) and reduced phosphorylation of YAP (Figure 3A, left panels). In order to assess whether SEMA6A/RhoA/YAP induction by BRAF and MEK inhibition specifically occurs in a BRAF-mut context, we administered same treatments to 2/17 and ME4405 (BRAF-wt/NRAS-mut), and ME1007 (BRAF-wt/NRAS-wt) cell lines. As expected, pERK were inhibited by trametinib and paradoxically induced by dabrafenib as previously described (46) in NRAS-mut cell lines (Figure 3A, central panels) and were affected only to a marginal extent in the BRAFwt/NRAS-wt ME1007 cells (Figure 3A, right most panel). The treatments did not induce SEMA6A expression, nor the levels of phosphorylated YAP were affected in the BRAF-wt cell lines analyzed (Figures $3 \mathrm{~A}$ right panels). These results indicate that BRAF inhibition by dabrafenib, MEK inhibition by trametinib, and dual BRAF/MEK inhibition by dabrafenib+trametinib induce the SEMA6A/RhoA/YAP axis in BRAF-mut but not in BRAF-wt melanoma cells.

To investigate the dynamics of SEMA6A/RhoA/YAP axis activation and actin cytoskeleton remodeling in response to BRAF and MEK inhibition, we treated BRAF-mut 2/59 and BRAF-wt ME4405 cells up to 7 days and analyzed them by immunofluorescence. Following 48 hours dabrafenib+trametinib, BRAF-mut 2/59 cells showed complete disorganization of actin cytoskeleton and YAP nuclear translocation (Figure 3D, left upper panels, and Figure 4A, left upper panels). Afterwards, surviving cells gradually re-organized their actin cytoskeleton, as shown by images taken 96 hours post treatment (Figure 3D, left middle panels), and rescued stress fibers/cytoskeleton organization and YAP cytoplasmic localization after 7 days treatment (Figure 3D, left lower panels and Figure 4A, left lower panels). Loss of stress fibers and YAP nuclear translocation were mainly induced in $2 / 59$ cells by dabrafenib and dabrafenib+trametinib combination, and only to a lesser extent by trametinib (Figure 4A, left upper panels). Figure 4B shows the increase in percentage of nuclear YAP upon 48 hours and 7 days trametinib $(34,4 \%$ and $22,2 \%)$, dabrafenib (81,6\% and $71,3 \%$ ) and their combination (95,2\% and $76,5 \%)$ compared with untreated cells ( $5 \%$ and $3,6 \%$ ). By contrast, in BRAF-wt ME4405 cells, the actin cytoskeleton was not affected by dual BRAF/MEK inhibition (Figure 4A, right panels), and trametinib only induced a mild YAP nuclear translocation (6,7\% at 48 hours and 7 days) (Figure $4 \mathrm{~A}$, right panels, and Figure $4 \mathrm{~B}$, right graphs). These 
data suggest that, in response to dabrafenib and dabrafenib+trametinib, BRAF-mut melanoma cells activate the axis SEMA6A/RhoA/YAP as a compensatory mechanism aimed to sustain actin cytoskeleton remodeling.

Next, we assessed the viability of shCtrl and shSEMA6A cells following drugs treatments. Dabrafenib and dabrafenib+trametinib treatments induced a marked reduction in the number of viable shCtrl cells (Supplementary Figure S1). As expected, SEMA6A depletion per se reduced the number of viable A3 and more efficiently that of $\mathrm{H} 2$ cells compared with shCtrl cells (Supplementary Figure S1, compare black bars of untreated cells); however, it did not further affect the number of viable cells following drug treatments (Supplementary Figure S1, compare grey bars of treated cells), suggesting that some other factors may be required for SEMA6A/RhoA/YAP axis to regulate sensitivity to targeted therapies in patients.

\section{SEMA6A is a mediator of surrounding fibrobalsts-induced melanoma cell growth.}

Sorrounding fibroblasts have been previously reported to promote the growth of melanoma cells in vitro (48); thus we asked whether microenvironment might play a role in SEMA6A signaling and exploited a coculture condition in which melanoma cells are cultured on a monolayer of BJ human fibroblasts. Fibroblasts-cocultured (from hereon, co-cultured) shCtrl had a markedly higher proliferation rate compared with the same cells cultured in the absence of fibroblasts (from hereon, mono-cultured). SEMA6A depletion nearly abolished the proliferative stimulus conferred by fibroblasts (Figures 5A); indeed, the fibroblasts-dependent fold growth induction was significantly reduced in SEMA6A-depleted cells compared to shCtrl cells (Figure 5B).

In addition, we sorted GFP-positive shCtrl and SEMA6A-depleted cell populations from co-cultures and analyzed the phosphorylation levels of YAP and of survival and proliferation effectors AKT, P65, and ERK in co-cultured and mono-cultured cells. In agreement with their higher proliferation rate, co-cultured shCtrl cells showed higher phosphorylation levels of YAP, AKT, P65, and ERK compared with their mono-cultured counterpart (Figure 5C). SEMA6A depletion increased the levels of phosphorylated and total YAP in mono-cultured condition, as expected, and also in co-cocultured cells. Of relevance, SEMA6A depletion reduced the phosphorylation levels of AKT, P65, and ERK only in the presence of sorrounding fibroblasts (Figure 5C).

These findings recapitulate previous evidence indicating that surrounding fibroblasts sustain the growth of melanoma cells by the activation of pro-survival (PI3K/AKT) and pro-proliferative (MAPK, NFkB) pathways and indicate that SEMA6A plays a crucial role as a mediator in this process.

\section{SEMA6A depletion rescues the efficacy of both BRAF and dual BRAF/MEK inhibition in fibroblasts- cocultured melanoma cells.}

Tumor microenvironment is known to increase resistance to BRAF inhibitors $(28,29)$. Since SEMA6A supports surrounding fibroblasts-induced growth of melanoma cells, we asked whether SEMA6A might affect the efficacy of targeted therapy in co-cultured condition. Thus, we treated mono-cultured and co- 
cultured shCtrl and SEMA6A-depleted A3 and H2 cell populations with dabrafenib or dabrafenib+trametinib combination, and periodically monitored cell viability over time up to 156 hours.

First, we observed reduced efficacy of dabrafenib in co-cultered cells. Indeed, as shown in Figure 5D, the proliferation rate of both mono-cultured and co-cultured shCtrl cells increased irrespective of treatments; dabrafenib rather conferred a paradoxical growth advantage in co-cultured condition (Figure 5D and 5E). Then, we found that in SEMA6A-depleted cells both dabrafenib and dabrafenib+trametinib treatments dramatically reduced cell proliferation only in the co-cultured condition (Figure 5F). As shown in Figure 5G, 156 hours post-dabrafenib and dabrafenib+trametinib, the number of co-cultured viable SEMA6Adepleted cells was significantly reduced compared with untreated cells. Data from co-cultured shCtrl and SEMA6A-depleted $\mathrm{A} 3$ and $\mathrm{H} 2$ cells reported as fold change number of treated/untreated viable cells show that SEMA6A depletion rescued the efficacy of both dabrafenib and dabrafenib+trametinib (Figure $5 \mathrm{H}$ ); indeed, 180 hours post-treatment the viability of treated/untreated SEMA6A-depleted cells was significantly reduced as compared with shCtrl cells (Figure 5I).

Next, we analyzed the levels of phosphorylated and total AKT, ERK and YAP of both shCtr and shSEMA6A cells mono-cultured and sorted from coculture, following dabrafenib+trametinib treatment. The combination treatment induced SEMA6A expression in mono-cultured shCtrl cells, as expected, and also in co-cultured shCtrl cells. Of relevance, upon dabrafenib+trametinib, SEMA6A depletion reduced the levels of phosphorylated AKT and ERK compared with treated shCtrl cells only in co-cultured condition (Figure 5L).

Overall these data indicate that surrounding fibroblasts protect shCtrl cells from the inhibitory effect of both BRAF and dual BRAF/MEK inhibition. SEMA6A depletion abrogates this protective effect and rescues the efficacy of both dabrafenib and dabrafenib+trametinib, indicating that SEMA6A plays a key role in mediating fibroblasts-induced insensitivity to targeted therapy.

\section{SEMA6A predicts progression free survival on dual BRAF/MEK inhibition in BRAF-mut melanoma.}

Based on the results we obtained in vitro, in the presence of surrounding fibroblasts as a microenvironment-mimicking condition, we investigated the predictive potential of SEMA6A on dual BRAF/MEK inhibition in vivo. To this aim, we analyzed the RECl2 cohort, which includes 14 BRAF-mut advanced melanoma patients admitted to dabrafenib+trametinib therapy at the Regina Elena Cancer Institute. Based on a cut-off value of 12 months of progression-free interval, eight of these patients were considered long-term responders and six short-term responders. Table 3 shows clinicopathological charachteristics of RECI2 cohort. SEMA6A expression was analyzed by IHC and reported both as percentage of positive cells and IS. Patients with high SEMA6A expression had a significantly reduced median PFS and OS compared with those with low SEMA6A (PFS: 10 months vs. 60 months; Log-Rank $p=0.001$; OS: 27.5 months vs. not reached; Log-Rank $p=0.021$ ) (Figures $6 \mathrm{~A}$ and $6 \mathrm{~B}$ ), indicating that SEMA6A might be a good candidate predictor of low efficacy of dual BRAF/MEK inhibition by dabrafenib+trametinib in BRAF-mut melanoma patients. 


\section{Discussion}

Melanoma is the most lethal form of skin cancer, with BRAF mutations occurring in about $50 \%$ of cases. Current targeted therapies include combinations of BRAF and MEK inhibitors and, among the approved ones, dabrafenib+trametinib is associated with a 5 -year survival rate of $34 \%$ (7). The introduction of immune checkpoint inhibitors (anti-CTLA4 and anti-PD1) has provided a further promising therapeutic option that improved outcomes in patients with metastatic melanoma (49-52) and the optimal sequence of targeted therapy and immunotherapy for the treatment of patients with BRAF-mut melanoma is under investigation in clinical trials (DREAMseq [NCT02224781] and SECOMBIT [NCT02631447]). In addition, the benefit of combining triplets of immune checkpoint inhibitors and targeted therapy is under evaluation in phase II and III clinical trials (53-55). With such a growing availability of therapeutic options, predictors of benefit from each class of drugs would be required in order to optimize the choice of treatment and improve patients outcomes.

In the present study, we provide evidence of the involvement of SEMA6A in the biology of BRAF-mut melanoma and the premise for its possible use as a predictive biomarker.

First, we show that SEMA6A protein expression is associated with BRAF mutational status in melanoma patients. SEMA6A has been shown to be deregulated together with many other cancer-related genes in the complex mechanism driven by $\mathrm{BRAF}^{\mathrm{V} 600 \mathrm{E}}$ on melanoma tumorigenesis (13); Guo and collegues also indicated the transcription factor MITF as a main mediator of BRAF ${ }^{\mathrm{V} 600 \mathrm{E}}$-driven transcription reprogramming. Moreover, a recent RNA-sequencing analysis conducted on SkMel-28 melanoma cells reveals a significant downregulation of SEMA6A following MITF knock out (56), suggesting the possible involvement of MITF in BRAF ${ }^{\mathrm{V} 600 \mathrm{E}}$-driven regulation of SEMA6A.

We then unravel the molecular mechanism by which SEMA6A regulates the remodeling of actin cytoskeleton thereby sustaining the aggressive behavior of BRAF-mut melanoma cells and show the involvement of downstream RhoA-YAP cascade. These findings are in agreement with previous reports showing that the increase in actin filaments promotes YAP nuclear translocation $(57,58)$. The functional cross-talk between actin cytoskeleton remodeling and YAP activity offers an explanation of how cells translate SEMA6A-induced cytoskeletal tension into their transcriptional program. Moreover, the identification of a SEMA6A-RhoA-YAP axis adds an important element of knowledge to the upstream regulation of YAP.

Second and to our opinion most relevant, we show that SEMA6A is involved in the mechanisms that regulate the sensitivity of BRAF-mut melanoma cells to dual BRAF/MEK inhibition therapy and acts as a crucial mediator of melanoma-stroma communication.

We show here that both dabrafenib and dabrafenib+trametinib induce the expression of SEMA6A and the downstream RhoA/YAP axis in vitro. SEMA6A induction by BRAF and MEK inhibition has been previously described $(59 ; 60)$, and higher SEMA6A expression has been reported in BRAF-inhibitor resistant cell lines as compared with sensitive ones (61). These findings support the role of this semaphorin as crucial 
player in the mechanisms of resistance to both BRAF and MEK inhibitors. In agreement, we show here that BRAF and dual BRAF/MEK inhibition cause in BRAF-mut melanoma cells an initial disorganization of actin cytoskeleton and the activation of SEMA6A/RhoA/YAP pathway; the resulting massive YAP nuclear translocation functionally determines rescue of actin cytoskeleton remodeling and survival.

However, our results indicate that surrounding fibroblasts play an essential role in coordinating evasion of co-cultured BRAF-mut melanoma cells from both BRAF and combined BRAF/MEK inhibition in a SEMA6A-dependent manner. Indeed, the expression of SEMA6A is associated with insensitivity to both treatments only in co-culturing condition, highlighting the role of SEMA6A as a critical transducer of signals from melanoma-stroma interactions. These data are in agreement with previous findings indicating that upon BRAF inhibition, melanoma-associated fibroblasts provide melanoma cells with drug tolerance (28), and co-culturing of melanoma cells with fibroblasts in vitro conveys protection to the growth inhibitory effects of the BRAF inhibitor vemurafenib (29). Among the reported mechanisms involved, are increased HGF release by host fibroblasts (26) and the promotion of matrix production and remodeling leading to elevated integrin b1/FAK/SRC signaling in melanoma cells (28). Moreover, a role of semaphorins in the regulation of microenvironment dynamic has been recently reported $(17,18)$. The expression of SEMA3B by cancer cells has been shown to recruit tumor-associated macrophages (TAMs) into the tumor microenvironment by the activation of a neuropilin-mediated signaling pathway that leads to an autocrine release of IL-8 (19), thereby promoting cancer progression. The production of SEMA4D by TAMs in the tumor stroma has been shown to sustain tumor angiogenesis and vessel maturation (20) while SEMA4D expressed by cancer cells sustains angiogenesis by binding plexin-B1 expressed by endothelial cells (21-23). Several semaphorins and plexins are expressed by dendritic cells and lymphocytes $(24,25)$, suggesting a role of these proteins in the immune response. Overall these evidences foster the role of semaphorins in instructing stroma cells to perform specific functions, mainly aimed at cancer progression. However there is no previous evidence of the role of SEMA6A in melanomamicroenvironment interactions.

Our in vitro results were strongly supported by data obtained from a cohort of BRAF-mut melanoma patients admitted to dabrafenib+trametinib treatment. Indeed, we showed that SEMA6A expression is associated with short-time benefit from dual BRAF/MEK inhibition. Despite a small number of patients was included in this cohort, they were homogeneously distributed between short-term and long-term responders and the association between SEMA6A expression and recurrence-free interval was highly significant and high SEMA6A expression was detected in all the short-term responders. Unfortunately, we could not extend our analysis in publicly available larger cohorts, due to unavailability of mRNA data from BRAF-mut patients admitted to dual BRAF/MEK targeted therapy. Thus, further investigation in larger patient cohorts will be required in order to assess the use of SEMA6A as predictive biomarker for dual BRAF/MEK targeted therapy.

\section{Conclusion}


Overall, our study indicates that SEMA6A is a crucial player in the biology of BRAF-mut melanoma, with high protein expression being associated with worse prognosis in term of OS and PFS of advanced disease and shorter relapse-free interval of early disease. Mechanistically, SEMA6A activates a RhoA/YAP axis that functionally results in actin cytoskeleton remodeling. Furthermore, dual BRAF/MEK inhibition induces early actin cytoskeleton disruption and activates SEMA6A/RhoA/YAP signaling that finally results in rescue of actin cytoskeleton and reduced efficacy of targeted therapy in a microenvironmentmimicking culture condition. Finally, SEMA6A protein expression predicts shorter recurrence-free interval in patients treated with dual BRAF/MEK inhibition. Thus, the possible use of SEMA6A protein assessment as predictive biomarker in BRAF-mut melanoma deserves further investigation.

\section{Abbreviations}

SEMA6A: Semaphorin 6A; RhoA: Ras homolog family member A; YAP: Yes-associated protein; BRAF: BRAF proto-oncogene, serine/threonine kinase; NRAS: NRAS proto-oncogene, GTPAase; MEK: Mitogenactivated protein kinase kinase; MAPK: Mitogen-activated protein kinase; RECl: Regina Elena Cancer Institute; TCGA: The cancer genome atlas; DFCl: Dana-Farber Cancer Institute; OS: Overall survival; PFS: Progression-free survival; RFS: Relapse-free survival; CAFs: Cancer-associated fibroblasts; IHC: Immunohistochemistry; IS: Intensity score; GFP: Green fluorescent protein; MITF: Melanocyte inducing transcription factor; TAMs: tumor-associated macrophages; WB: Western blot

\section{Declarations}

\section{Acknowledgements}

We thank Giulia Federici for her technical support.

\section{Authors' contributions}

Giulia Bon, Rita Falcioni and Rossella Loria designed the experiments. Rossella Loria performed the main experiments. Valentina Laquintana and Paolo Visca addressed the Immunohistochemistry study. Stefano Scalera performed the bioinformatics analyses. Rocco Fraioli, Valentina Caprara, Italia Falcone, Chiara Bazzichetto, Marta Di Martile, Laura Rosanò, Donatella Del Bufalo and Gianluca Bossi contributed to the experiments. Isabella Sperduti and Irene Terrenato performed the statistical analyses. Virginia Ferraresi, Gennaro Ciliberto, Michele Milella, and Silvia Soddu made a critical revision. Giulia Bon, Rossella Loria and Rita Falcioni analyzed the data and wrote the manuscript. All the authors read and approved the final manuscript.

\section{Funding}

This work was supported by grant AIRC IG 18622 (to MM and RF).

\section{Availability of data and materials}


The datasets used and/or analyzed during the current study are available from the corresponding author on reasonable request.

\section{Ethics approval and consent to participate}

The study was conducted in accordance with the Declaration of Helsinki.

\section{Consent for publication}

All of the authors are aware of and agree to the content of the paper and their being listed as a co-author of the paper.

\section{Competing interests}

VF has received speaker fees/advisory boards from Novartis, Bristol Meyers Squibb, MSD and Pierre Fabre, not related to the topic of this manuscript. MM has received honoraria and consultancy fees from Novartis, not related to the topic of this manuscript. The remaining authors declare no competing financial interests.

\section{References}

1. Lawrence MS, Stojanov P, Polak P, Kryukov GV, Cibulskis K, Sivachenko A, et al. Mutational heterogeneity in cancer and the search for new cancer-associated genes. Nature. 2013;499:214-8.

2. Fattore L, Mancini R, Acunzo M, Romano G, Laganà A, Pisanu ME, et al. miR-579-3p controls melanoma progression and resistance to target therapy. P Natl Acad Sci USA. 2016;8:E5005-13.

3. Mannavola F, Tucci M, Felici C, Passarelli A, D'Oronzo S and Silvestri F. Tumor-derived exosomes promote the in vitro osteotropism of melanoma cells by activating the SDF-1/CXCR4/CXCR7 axis. J Transl Med. 2019;17:230.

4. Dror S, Sander L, Schwartz H, Sheinboim D, Barzilai A, Dishon Y, et al. Melanoma miRNA trafficking controls tumour primary niche formation. Nat Cell Biol. 2016;18:1006-17.

5. Hunter Shain A, Yeh I, Kovalyshyn I, Sriharan A, Talevich E, Gagnon A, et al. The genetic evolution of melanoma from precursor lesions. N Engl J Med. 2015;373:1926-36.

6. Chapman PB, Hauschild A, Robert C, Haanen JB, Ascierto P, Larkin J, et al. Improved survival with vemurafenib in melanoma with BRAF V600E mutations. N Engl J Med. 2011;364:2507-16.

7. Robert C, Grob JJ, Stroyakovskiy D, Karaszewska B, Hauschild A, Levchenko E, et al. Five-Year Outcomes with Dabrafenib plus Trametinib in Metastatic Melanoma. N Engl J Med. 2019;381:62636.

8. Long GV, Stroyakovskiy D, Gogas H, Levchenko E, de Braud F, Larkin J, et al. Combined BRAF and MEK Inhibition versus BRAF Inhibition Alone in Melanoma. N Engl J Med. 2014;371:1877-88.

9. Long GV, Flaherty KT, Stroyakovskiy D, Gogas H, Levchenko E, de Braud F, et al. Dabrafenib plus trametinib versus dabrafenib monotherapy in patients with metastatic BRAF V600E/ K-mutant 
melanoma: long-term survival and safety analysis of a phase 3 study. Ann Oncol. 2017;28:1631-9.

10. Robert C, Karaszewska B, Schachter J, Rutowski P, Mackiewicz A, Stryakovskiy D, et al. Two year estimate of overall survival in COMBI-v, a randomized, open-label, phase III study comparing the combination of dabrafenib (D) and trametinib (T) with vemurafenib (vem) as first-line therapy inpatients (pts) with unresectable or metastatic BRAF V600E/K mutation-positive cutaneous melanoma. Eur J Cancer. 2015; 51(Suppl. 3):S720-3.

11. Ascierto PA, Ribas A, Larkin J, McArthur GA, Lewis KD, Hauschild A, et al. Prognostic subgroups and impact of treatment for post-progression overall survival (ppOS) in patients (pts) with BRAFV600mutated metastatic melanoma treated with dacarbazine (DTIC) or vemurafenib (VEM) \pm cobimetinib (COBI): A pooled analysis. Ann Oncol. 2016; 27(Suppl. 6):vi394.

12. Loria R, Bon G, Baldassarri P, Gallo E, Bersani I, Porru M, et al. Sema6A and Mical1 in metastatic BRAFV600E mutant melanomas: novel targets for therapy. Oncotarget. 2014;6:2779-93.

13. Guo $X, X u Y$ and Zhao Z. In-depth genomic data analyses revealed complex transcriptional and epigenetic dysregulations of BRAF ${ }^{\mathrm{V} 600 \mathrm{E}}$ in melanoma. Mol Cell. 2015;14:60.

14. Tamagnone L. Emerging role of semaphorins as major regulatory signals and potential therapeutic targets in cancer. Cancer Cell. 2012;22:145-52.

15. Gurrapu S and Tamagnone L. Transmembrane semaphorins: Multimodal signaling cues in development and cancer. Cell Adh Migr. 2016,10(6):675-91.

16. Valentini E, Di Martile M, Del Bufalo D and D'Aguanno S. SEMAPHORINS and their receptors: focus on the crosstalk between melanoma and hypoxia. J Exp Clin Cancer Res. 2021;40:131.

17. Capparuccia $L$ and Tamagnone $L$. Semaphorin signaling in cancer cells and in cells of the tumor microenvironment - two sides of a coin. J Cell Sci. 2009;122:1723-36.

18. Gu C and Giraudo E. The role of semaphorins and their receptors in vascular development and cancer. Exp Cell Res. 2013;319(9):1306-16.

19. Rolny C, Capparuccia L, Casazza A, Mazzone M, Vallario A, Cignetti A, et al. The tumor suppressor semaphorin 3B triggers a prometastatic program mediated by interleukin 8 and the tumor microenvironment. J Exp Med. 2008;205:1155-71.

20. Sierra JR, Corso S, Caione L, Cepero V, Conrotto P, Cignetti A, et al. Tumor angiogenesis and progression are enhanced by Sema4D produced by tumor-associated macrophages. J Exp Med. 2008;205:1673-85.

21. Basile JR, Barac A, Zhu T, Guan KL and Gutkind JS. Class IV semaphorins promote angiogenesis by stimulating Rho-initiated pathways through plexin-B. Cancer Res. 2004;64:5212-24.

22. Basile JR, Afkhami T and Gutkind JS. Semaphorin 4D/plexin-B1 induces endothelial cell migration through the activation of PYK2, Src, and the phosphatidylinositol 3-kinase-Akt pathway. Mol Cell Biol. 2005;25:6889-98.

23. Conrotto P, Valdembri D, Corso S, Serini G, Tamagnone L, Comoglio PM, et al. Sema4D induces angiogenesis through Met recruitment by Plexin B1. Blood. 2005;105:4321-9. 
24. O'Connor BP and Ting JP. The evolving role of semaphorins and plexins in the immune system: Plexin-A1 regulation of dendritic cell function. Immunol Res. 2008;41:217-22.

25. Suzuki K, Kumanogoh A and Kikutani H. Semaphorins and their receptors in immune cell interactions. Nat Immunol. 2008;9:17-23.

26. Straussman R, Morikawa T, Shee K, Barzily-Rokni M, Qian ZR, Du J, et al. Tumour micro-environment elicits innate resistance to RAF inhibitors through HGF secretion. Nature. 2012;487(7408):500-4.

27. Zhou L, Yang K, Andl T, Wickett RR and Zhang Y. Perspective of Targeting Cancer-Associated Fibroblasts in Melanoma. J Cancer. 2015;6(8):717-26.

28. Hirata E, Girotti MR, Viros A, Hooper S, Spencer-Dene B, Matsuda M, et al. Intravital imaging reveals how BRAF inhibition generates drug-tolerant microenvironments with high integrin $\beta 1 / F A K$ signaling. Cancer Cell. 2015;27(4):574-88.

29. Fedorenko IV, Wargo JA, Flaherty KT, Messina JL and Smalley KSM. BRAF Inhibition Generates a Host-Tumor Niche that Mediates Therapeutic Escape. J Invest Dermatol. 2015;135(12):3115-24.

30. Butcher DT, Alliston T and Weaver VM. A tense situation: forcing tumour progression. Nat Rev Cancer. 2009;9:108-22.

31. Paszek MJ, Zahir N, Johnson KR, Lakins JN, Rozenberg GI, Gefen A, et al. Tensional homeostasis and the malignant phenotype. Cancer Cell. 2005;8:241-54.

32. Dupont S, Morsut L, Aragona M, Enzo E, Giulitti S, Cordenonsi M, et al. Role of YAP/TAZ in mechanotransduction. Nature. 2011;474:179-83.

33. Zhou D, Conrad C, Xia F, Park JS, Payer B, Yin Y, et al. Mst1 and Mst2 maintain hepatocyte quiescence and suppress hepatocellular carcinoma development through inactivation of the Yap1 oncogene. Cancer Cell. 2009;16(5):425-38.

34. Nallet-Staub F, Marsaud V, Li L, Gilbert C, Dodier S, Bataille V, et al. Pro-invasive activity of the Hippo pathway effectors YAP and TAZ in cutaneous melanoma. J Invest Dermatol. 2014;134(1):123-32.

35. Zhao B, Lei Q-Y and Guan K-L. The Hippo-YAP pathway: new connections between regulation of organ size and cancer. Curr Opin Cell Biol. 2008;20(6):638-46.

36. Bao Y, Hata Y, Ikeda M and Withanage K. Mammalian Hippo pathway: from development to cancer and beyond. J Biochem. 2011;149(4):361-79.

37. Chan SW, Lim CJ, Chen L, Chong YF, Huang C, Song H, et al. The Hippo pathway in biological control and cancer development. J Cell Physiol. 2011;226(4):928-39.

38. Yin M and Zhang L. Hippo signaling: A hub of growth control, tumor suppression and pluripotency maintenance. J Genet Genomics. 2011;38(10):471-81.

39. Varelas $X$ and Wrana JL. Coordinating developmental signaling: novel roles for the Hippo pathway. Trends Cell Biol. 2012;22(2):88-96.

40. Noto A, De Vitis C, Pisanu ME, Roscilli G, Ricci G, Catizone A, et al. Stearoyl-CoA-desaturase 1 regulates lung cancer stemness via stabilization and nuclear localization of YAP/TAZ. Oncogene. 2017;32:4573-84. 
41. Shen T, Li Y, Zhu S, Yu J, Zhang B, Chen X, et al. YAP1 plays a key role of the conversion of normal fibroblasts into cancer-associated fibroblasts that contribute to prostate cancer progression. J Exp Clin Cancer Res. 2020;39:36.

42. Pisanu ME, Maugeri-Saccà M, Fattore L, Bruschini S, De Vitis $C$, Tabbì E, et al. Inhibition of StearoylCoA desaturase 1 reverts BRAF and MEK inhibition-induced selection of cancer stem cells in BRAFmutated melanoma. J Exp Clin Cancer Res. 2018;37:318.

43. Fattore L, Mancini R, and Ciliberto G. Cancer Stem Cells and the Slow Cycling Phenotype: How to Cut the Gordian Knot Driving Resistance to Therapy in Melanoma. Cancers. 2020;12:3368.

44. Kim MH, Kim J, Hong H, Lee SH, Lee JK, Jung E, et al. Actin remodeling confers BRAF inhibitor resistance to melanoma cells through YAP/TAZ activation. EMBO J. 2016;35:462-78.

45. Lin L, Sabnis AJ, Chan E, Olivas V, Cade L, Pazarentzos E, et al. The Hippo effector YAP promotes resistance to RAF- and MEK- targeted cancer therapies. Nat Genet. 2015;47(3):250-6.

46. Sharma S, Santiskulvong C, Rao J, Gimzewski JK, and Dorigo O. The role of Rho GTPase in cell stiffness and cisplatin resistance in ovarian cancer cells. Integr Biol. 2014; 6(6):611-7.

47. Hatzivassiliou G, Song K, Yen I, Brandhuber BJ, Anderson DJ, Alvarado R, et al. RAF inhibitors prime wild-type RAF to activate the MAPK pathway and enhance growth. Nature. 2010;464:431-6.

48. Cornil I, Theodorescu D, Man S, Herlyn M, Jambrosic J and Kerbel RS. Fibroblast cell interactions with human melanoma cells affect tumor cell growth as a function of tumor progression. P Natl Acad Sci USA. 1991;88:6028-32.

49. Weber JS, D'Angelo SP, Minor D, Hodi FS, Gutzmer R, Neyns B, et al. Nivolumab versus chemotherapy in patients with advanced melanoma who progressed after anti-CTLA-4 treatment (CheckMate 037): a randomised, controlled, open-label, phase 3 trial. Lancet Oncol. 2015;16(4):375e84.

50. Robert C, Schachter J, Long GV, Arance A, Grob JJ, Mortier L, et al. Pembrolizumab versus ipilimumab in advanced melanoma. N Engl J Med. 2015;372(26):2521e32.

51. Hodi FS, Chesney J, Pavlick AC, Robert C, Grossmann KF, McDermott DF, et al. Combined nivolumab and ipilimumab versus ipilimumab alone in patients with advanced melanoma: 2-year overall survival outcomes in a multicentre, randomised, controlled, phase 2 trial. Lancet Oncol. 2016;17(11):1558e68.

52. Ascierto PA, McArthur GA, Dreno B, Atkinson V, Liszkay G, Di Giacomo AM, et al. Cobimetinib combined with vemurafenib in advanced BRAFV600-mutant melanoma (coBRIM): updated efficacy results from a randomised, double-blind, phase 3 trial. Lancet Oncol. 2016;17(9):1248e60.

53. Ferrucci PF, Di Giacomo AM, Del Vecchio M, Atkinson V, Schmidt H, Schachter J, et al. KEYNOTE-022 part 3: a randomized, double-blind, phase 2 study of pembrolizumab, dabrafenib, and trametinib in BRAF-mutant melanoma. J Immunother Cancer. 2020;8(2):e001806.

54. Gutzmer R, Stroyakovskiy D, Gogas H, Robert C, Lewis K, Protsenko S, et al. Atezolizumab, vemurafenib, and cobimetinib as first-line treatment for unresectable advanced BRAF ${ }^{\mathrm{V} 600 \mathrm{E}}$ mutationpositive melanoma (IMspire150): primary analysis of the randomised, double-blind, placebocontrolled, phase 3 trial. Lancet. 2020;395:1835-44. 
55. Dummer R, Lebbé $C$, Atkinson V, Mandalà $M$, Nathan PD, Arance A, et al. Combined PD-1, BRAF and MEK inhibition in advanced BRAF-mutant melanoma: safety run-in and biomarker cohorts of COMBIi. Nat Med. 2020;26(10):1557-63.

56. Dilshat R, Fock V, Kenny C, Gerritsen I, Lasseur RMJ, Travnickova J, et al. MITF reprograms the extracellular matrix and focal adhesion in melanoma. eLife. 2021;10:e63093.

57. Zhao B, Li L, Lu Q, Wang LH, Liu CY, Lei Q, et al. Angiomotin is a novel Hippo pathway component that inhibits YAP oncoprotein. Genes Dev. 2011;25(1):51-63.

58. Mana-Capelli S, Paramasivam M, Dutta S, and McCollum D. Angiomotins link F-actin architecture to Hippo pathway signaling. Mol Biol Cell. 2014;25(10):1676-85.

59. Parmenter TJ, Kleinschmidt M, Kinross KM, Bond ST, Li J, Kaadige MR, et al. Response of BRAFMutant Melanoma to BRAF Inhibition Is Mediated by a Network of Transcriptional Regulators of Glycolysis. Cancer Discov. 2014;4(4):423-33.

60. Ciuffreda L, Del Bufalo D, Desideri M, Di Sanza C, Stoppacciaro A, Ricciardi MR, et al. GrowthInhibitory and Antiangiogenic Activity of the MEK Inhibitor PD0325901 in Malignant Melanoma with or without BRAF Mutations. Neoplasia. 2009;11(8):720-31.

61. Ahn J-H, Hwang S-H, Cho H-S and Lee M. Differential Gene Expression Common to Acquired and Intrinsic Resistance to BRAF Inhibitor Revealed by RNA-Seq Analysis. Biomol Ther. 2019;27(3):30210.

\section{Tables}

Table 1. Clinicopathological characteristics of metastatic advanced melanoma patients from the RECI1 cohort ( $N=112)$. 


\begin{tabular}{|ll|}
\hline Characteristics & $\mathbf{N}(\%)$ \\
\hline Age yr, median (range) & $57(25-80)$ \\
\hline Gender & $55(49.1)$ \\
Male & $57(50.9)$ \\
Female & \\
\hline BRAF status & $59(52.7)$ \\
mut & $53(47.3)$ \\
wt & \\
\hline SEMA6A & $76(67,9)$ \\
Low & $36(32,1)$ \\
High & \\
\hline Metastatic Site & $55(49.1)$ \\
Lymph Node & $30(26.8)$ \\
Cutaneous/subcutaneous & $14(12.5)$ \\
Lung & $6(5.4)$ \\
Brain & $3(2.7)$ \\
Liver & $4(3.6)$ \\
Other & \\
\hline
\end{tabular}

Table 2. Progressively increasing expression of SEMA6A in subsequent metastatic lesions from three patients.

\begin{tabular}{|lllll|}
\hline & Diagnosis & & Progression \\
\hline Patient & $\%$ of SEMA6A+ cells & Tumor site & $\%$ of SEMA6A+ cells & Tumor site \\
\hline 1 & $60 \%$ & Back & $70 \%$ & Back \\
\hline 2 & $30 \%$ & Lung & $80 \%$ & Lung \\
\hline 3 & $10 \%$ & Leg & $70 \%$ & Occipital fragment \\
\hline
\end{tabular}


Table 3. Clinicopathological characteristics of $\mathrm{RECl} 2$ patients $(\mathrm{N}=14)$. 


\begin{tabular}{|c|c|c|c|c|c|c|}
\hline \multicolumn{7}{|c|}{ Short-Term Responders } \\
\hline Patient & Gender & $\begin{array}{l}\text { Progression-free } \\
\text { interval, mo }\end{array}$ & $\begin{array}{l}\text { OS, } \\
\text { mo }\end{array}$ & $\begin{array}{l}\text { \% of } \\
\text { SEMA6A+ cells }\end{array}$ & $\begin{array}{l}\text { SEMA6A } \\
\text { IS }\end{array}$ & Tumor sit \\
\hline 1 & $\mathrm{~F}$ & 12 & 35 & $70 \%$ & 2 & Metastasis \\
\hline 4 & M & 9 & 16 & $60 \%$ & 2 & Lung \\
\hline \multirow[t]{2}{*}{10} & & 11 & 18 & $60 \%$ & 2 & Lymph node \\
\hline & $\mathrm{F}$ & & & $50 \%$ & 1 & Lung \\
\hline 13 & $\mathrm{~F}$ & 4 & 20 & $60 \%$ & 2 & $\begin{array}{l}\text { Lung and lymph } \\
\text { node }\end{array}$ \\
\hline 14 & $\mathrm{~F}$ & 8 & 98 & $70 \%$ & 2 & $\begin{array}{l}\text { Occipital } \\
\text { fragment }\end{array}$ \\
\hline 15 & $\mathrm{~F}$ & 12 & 27 & $50 \%$ & 1 & Lung \\
\hline \multicolumn{7}{|c|}{ Long-Term Responders } \\
\hline Patient & Gender & $\begin{array}{l}\text { Progression-free } \\
\text { interval, mo }\end{array}$ & $\begin{array}{l}\text { OS, } \\
\text { mo }\end{array}$ & $\begin{array}{l}\% \text { of } \\
\text { SEMA6A+ cell }\end{array}$ & $\begin{array}{l}\text { SEMA6A } \\
\text { IS }\end{array}$ & Tumor site \\
\hline \multirow[t]{2}{*}{2} & M & Ongoing & 68 & $30 \%$ & 1 & Middle meatus \\
\hline & & 67 & & & & \\
\hline \multirow[t]{2}{*}{3} & $\mathrm{~F}$ & Ongoing & 91 & $30 \%$ & 1 & Lymph node \\
\hline & & 58 & & & & \\
\hline 5 & M & 39 & 63 & $60 \%$ & 2 & Back \\
\hline 6 & $M$ & 24 & 50 & $30 \%$ & 1 & Lung \\
\hline 7 & $M$ & 60 & 75 & $30 \%$ & 2 & Face \\
\hline \multirow[t]{2}{*}{8} & $M$ & Ongoing & 83 & $30 \%$ & 2 & Back \\
\hline & & 82 & & & & \\
\hline 9 & $M$ & 48 & 86 & $30 \%$ & 2 & Lung \\
\hline
\end{tabular}


11

F Ongoing

80

$30 \%$

1

Lymph node

75

Figures

Figure 1

A

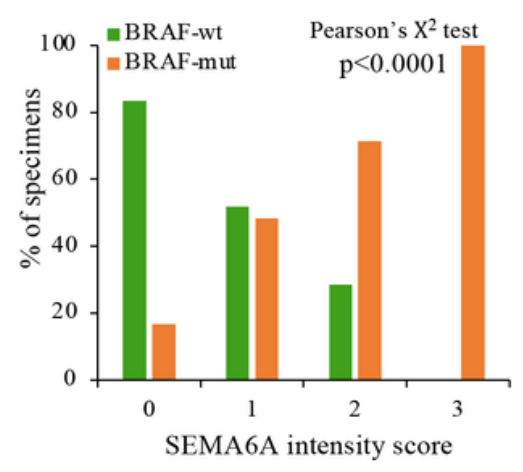

B

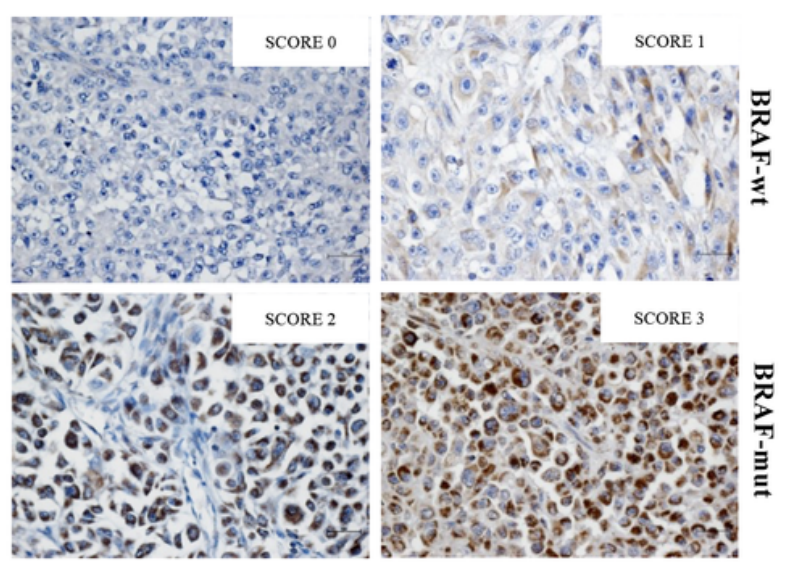

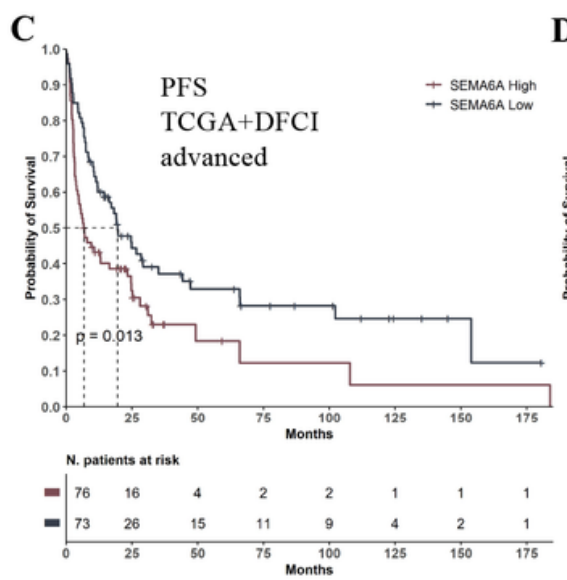

D

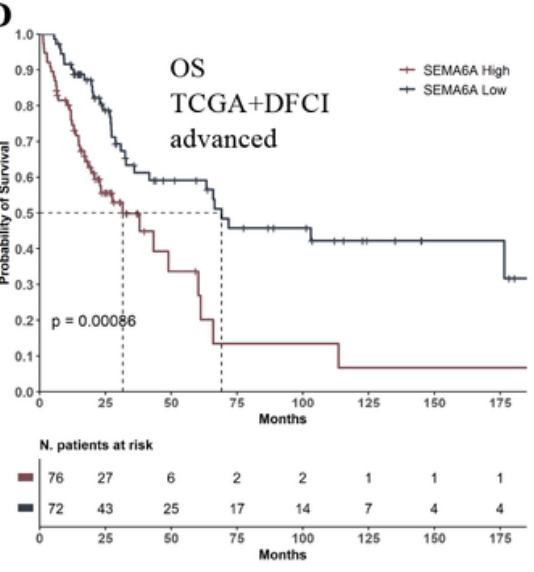

E

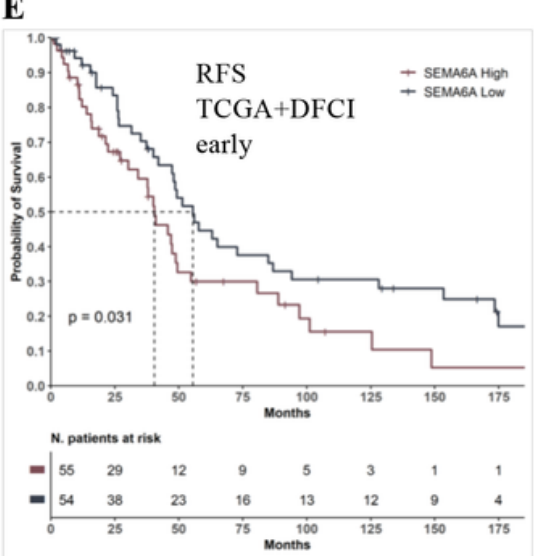

Figure 1 
SEMA6A is a prognostic indicator in BRAF-mut melanoma patients. SEMA6A Immunohistochemical assessment in BRAF-wt and BRAF-mut melanoma specimens of RECl1 cohort. Pearson's Chi-square test revealed a significant association between SEMA6A expression and BRAF mutation $(p<0,0001)(A)$.

Representative sections from 2 BRAF-wt and 2 BRAF-mut melanoma patients, stained with anti-SEMA6A antibody (B). Progression-free Survival (PFS) (C) and Overall Survival (OS) (D) in patients with with high and low SEMA6A (BRAF-mut advanced melanoma) and relapse-free survival (RFS) (E) in patients with with high and low SEMA6A (BRAF-mut early melanoma) from the cohorts TCGA and DFCl.

Figure 2

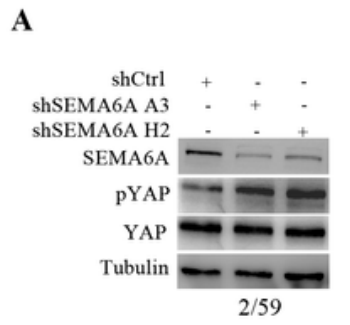

D
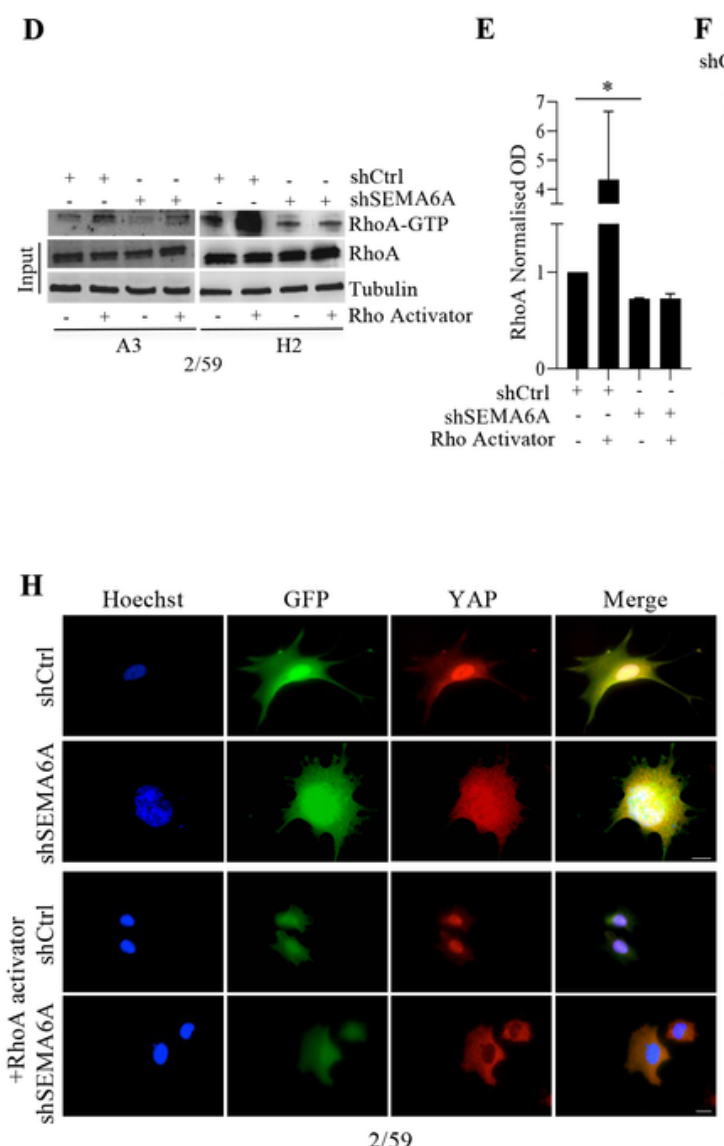

F
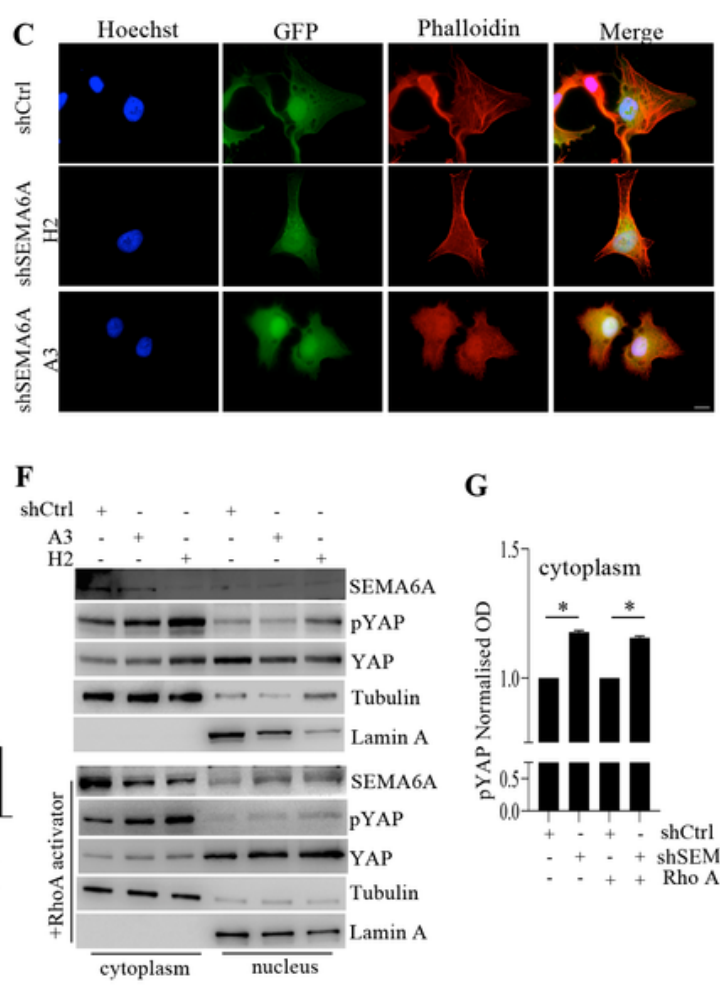

G
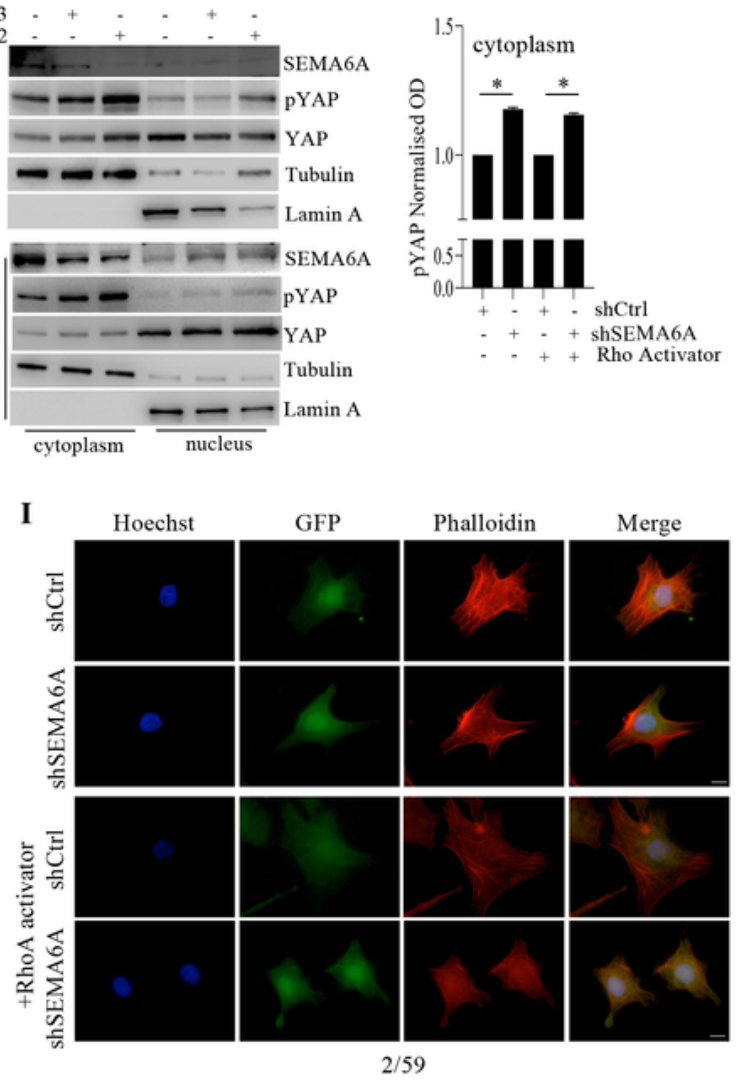


\section{Figure 2}

SEMA6A depletion reduces RhoA activity and induces YAP phosphorylation and cytoplasmic retention in BRAF-mut melanoma cells. Western Blot (WB) analysis of SEMA6A, phosphorylated and total YAP was performed on total cell extracts from inducible shCtrl and shSEMA6A A3 and H2 2/59 cells upon silencing induction. The anti-tubulin antibody was used to validate equivalent amount of loaded proteins in each lane (A). Fold change number of viable $\mathrm{A} 3$ and $\mathrm{H} 2$ cells compared with shCtrl cells 72 hours postinduction (B). shCtr and SEMA6A-depleted $\mathrm{A} 3$ and $\mathrm{H} 2$ cells were plated on poly-l lysine coated slides and stained with Phalloidin (red signal) (C). GFP reporter gene expression revealed successful silencing induction. The cells were counterstained with Hoechst to highlight nuclei. Scale bar $10 \mu \mathrm{m}$. WB analysis of activated RhoA (RhoA-GTP) pulled down from cell lysates and total RhoA on cell extracts from shCtrl and SEMA6A-depleted 2/59 cells, treated or not with $1 \mathrm{U} / \mathrm{mL}$ RhoA activator. The anti-tubulin antibody was used to validate equivalent amount of loaded proteins in each lane (D). Data from three independent experiments were analyzed by densitometric analysis of RhoA-GTP normalized to RhoA. Data obtained from SEMA6A-depleted $\mathrm{A} 3$ and $\mathrm{H} 2$ cell populations were pulled and indicated as shSEMA6A. The results are presented as mean $+/$ - standard deviation of three independent experiments $\left({ }^{*} p<0.05\right)$. $(E)$.

Cytoplasmic and nuclear fractions extracted from shCtr and $\mathrm{A} 3$ and $\mathrm{H} 2$ cells, treated or not with RhoA activator, were analyzed by WB for the expression of SEMA6A, phosphorylated and total YAP. Lamin A and a-tubulin were used to validate purity of nuclear and cytoplasmic extracts respectively ( $F$ ). Data from three independent experiments were analyzed by densitometric analysis of p-YAP normalized to total YAP in cytoplasmic fraction (G). shCtr and SEMA6A-depleted cells plated on poly-l lysine coated slides were treated or not with RhoA activator and stained with anti-YAP (red signal) $(\mathrm{H})$, or with Phalloidin (red signal) (I). Scale bar $10 \mu \mathrm{m}$. 
Figure 3
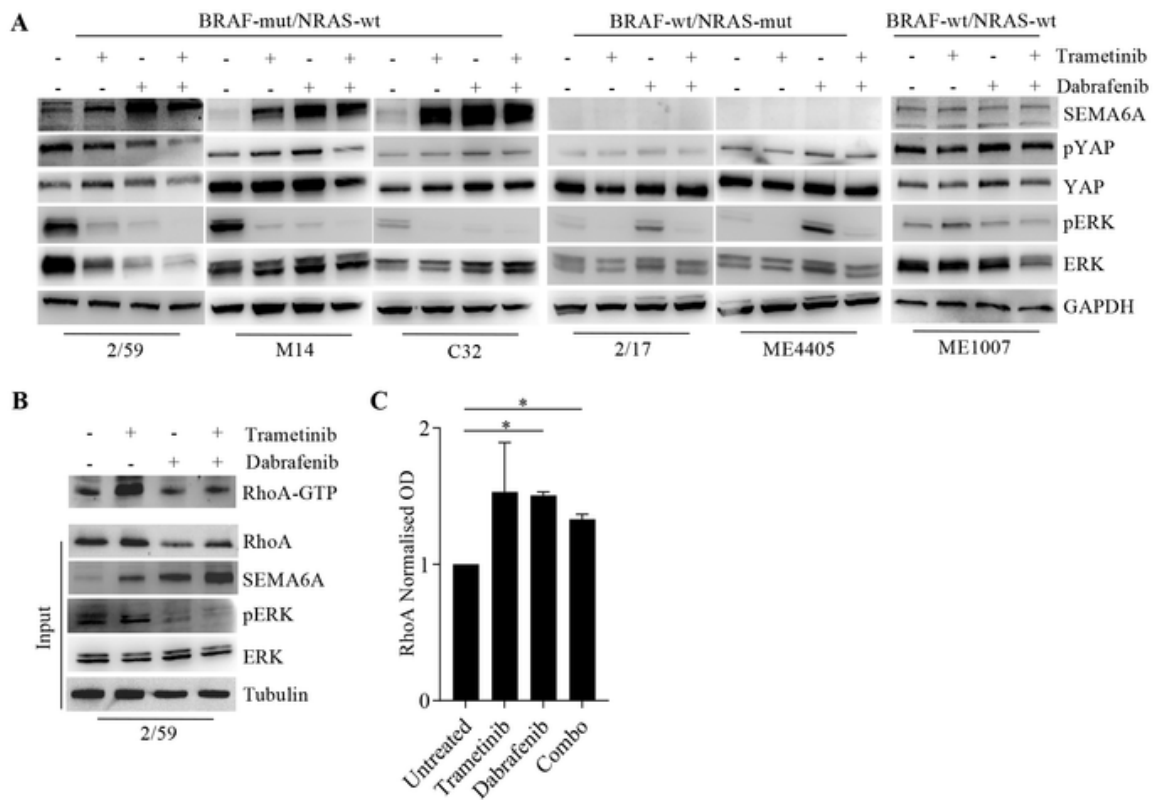

D
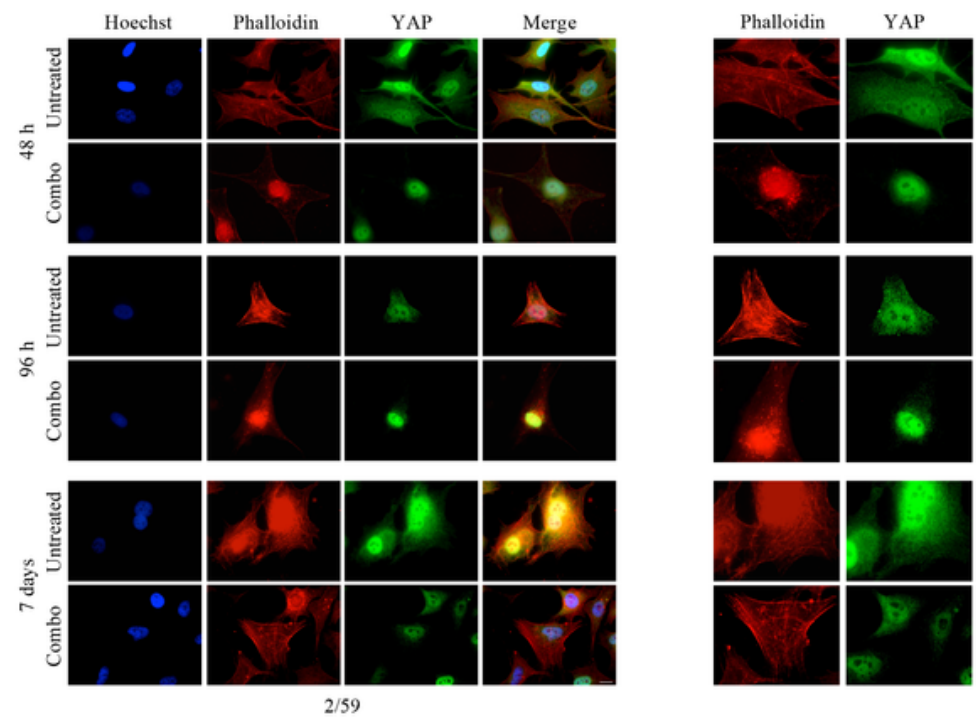

\section{Figure 3}

Dabrafenib, trametinib, and their combination induce SEMA6A-RhoA-YAP axis in BRAF-mut melanoma cells. BRAF-mut/NRAS-wt 2/59, M14 and C32, BRAF-wt/NRAS-mut 2/17 and ME4405, and BRAFwt/NRAS-wt ME1007 cell lines were treated with 0,1 $\mu \mathrm{M}$ dabrafenib, 5nM trametinb and their combination for 48 hours. Western Blot (WB) analysis of SEMA6A, phosphorylated and total YAP and ERK was performed on total cell extracts from untreated and treated cells. The anti-GAPDH antibody was used to 
validate equivalent amount of loaded proteins in each lane (A). WB analysis of activated RhoA (RhoAGTP) pulled down from cell lysates and RhoA, SEMA6A, phosphorylated and total ERK on total cell extracts from untreated and treated 2/59 cells as specified. The anti-tubulin antibody was used to validate equivalent amount of loaded proteins in each lane (B). Densitometric analysis of RhoA-GTP normalized to RhoA is reported (C). The results are presented as mean+/- standard deviation of three independent experimets $\left({ }^{\star} p<0.05\right)$. BRAF-mut $2 / 59$ cells plated on poly-l lysine coated slides were treated or not with dabrafenib+trametinib for $48 \mathrm{~h}, 96 \mathrm{~h}$ and 7 days, and stained with anti-YAP (green signal) and Phalloidin (red signal). The cells were counterstained with Hoechst to highlight nuclei. Scale bar $10 \mu \mathrm{m}(\mathrm{D})$. Magnification of Phalloidin and YAP images is reported in right most panels. 
Figure 4
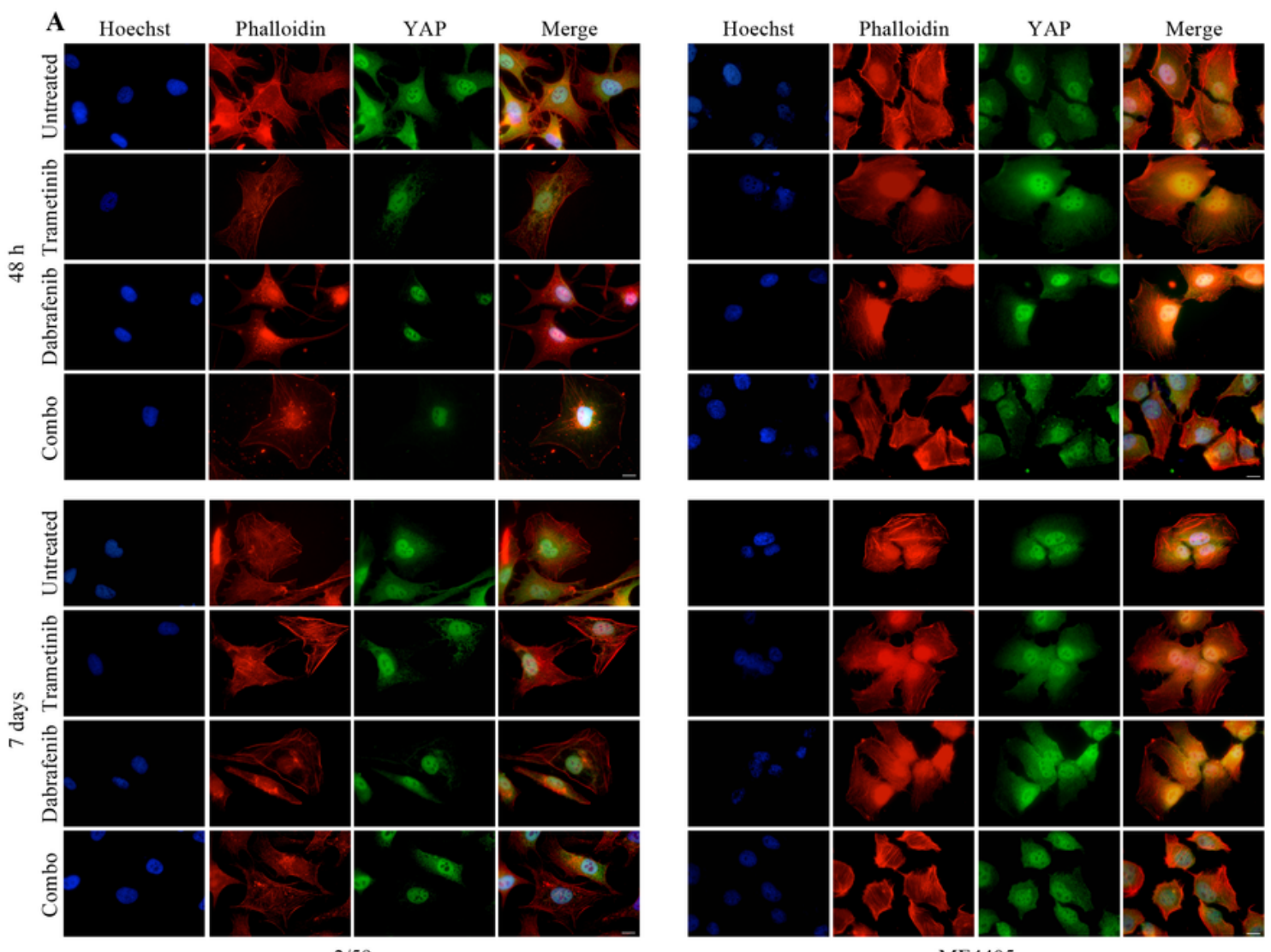

$2 / 59$

ME4405

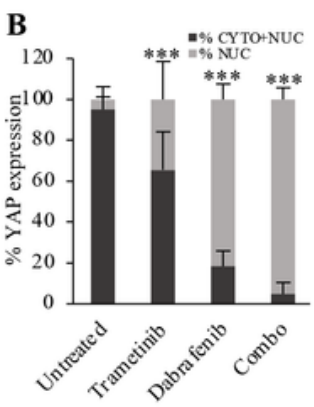

$48 \mathrm{~h}$

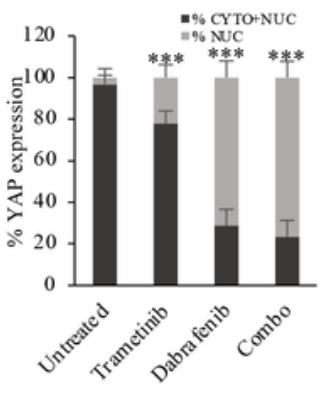

7 days

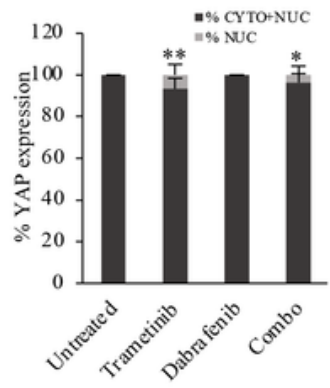

$48 \mathrm{~h}$

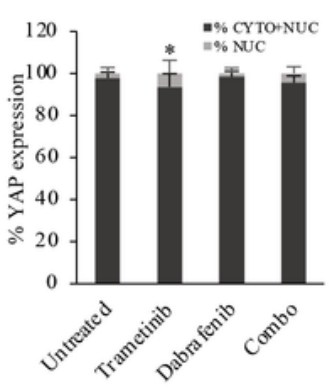

7 days

\section{Figure 4}

Dabrafenib- and dabrafenib+trametinib-induced SEMA6A-RhoA-YAP axis activation rescues actin cytoskeleton in BRAF-mut melanoma cells BRAF-mut 2/59 and BRAF-wt ME4405 cells plated on poly-I lysine coated slides were treated or not with dabrafenib, trametinib and their combination for $48 \mathrm{~h}$ and 7 days, and stained with anti-YAP (green signal) and Phalloidin (red signal). The cells were counterstained with Hoechst to highlight nuclei. Scale bar $10 \mu \mathrm{m}$ (A). Quantification of the subcellular localization of YAP 
from immunofluorescence of 2/59 and ME4405 cells untreated and treated with dabrafenib, trametinib and their combination for 48 hours and 7 days. The results are presented as mean+/- standard deviation of three independent experimets ( $\left.{ }^{*} p<0.05 ;{ }^{* \star} p<0,001 ; * \star * p<0,0001\right)(B)$.

\section{Figure 5}

A

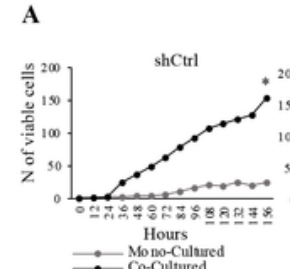
$\because$ - Mono-Cultured

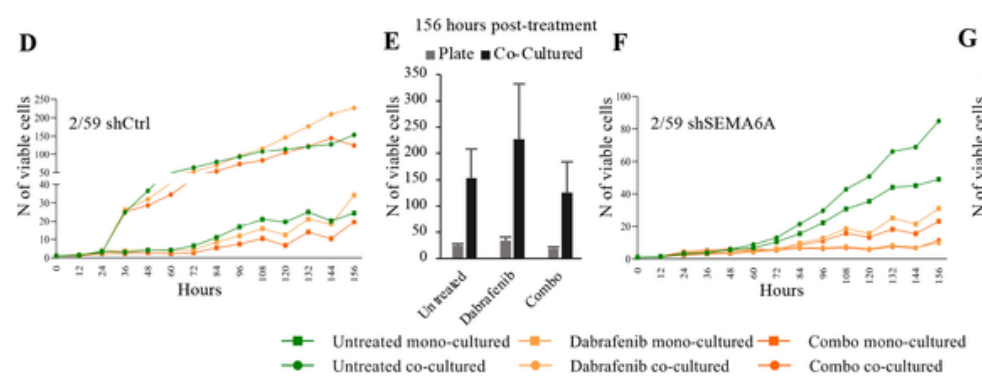

C

G 156 hours post-treatment $M=$ Plate $\mathbf{m}-$ Coultured

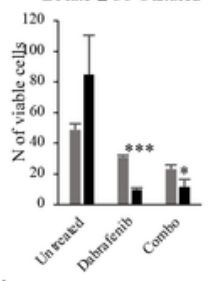

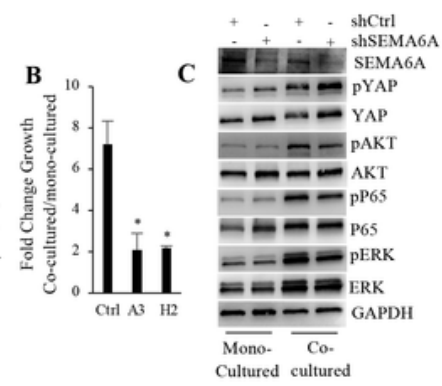
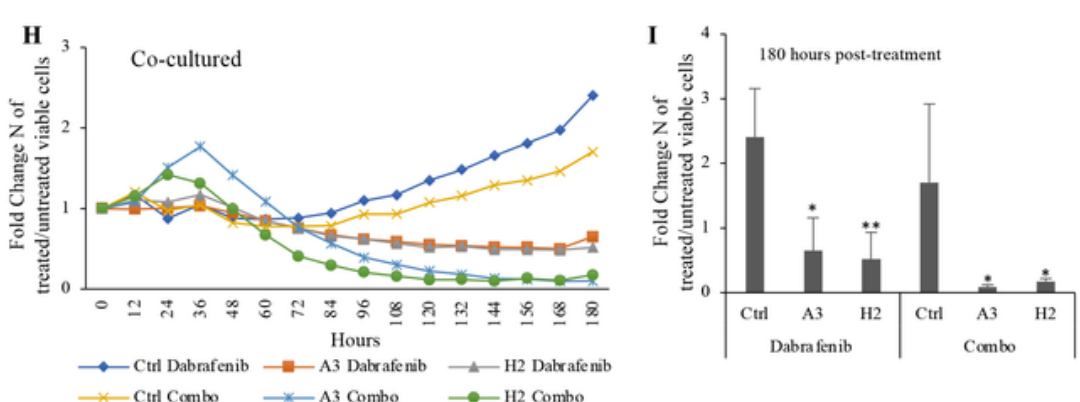

L

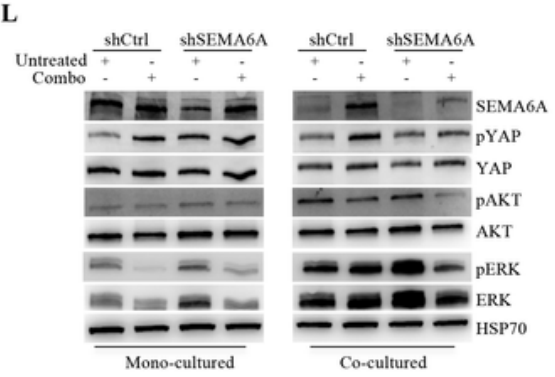

\section{Figure 5}

SEMA6A depletion restores responsiveness to dabrafenib and dabrafenib+trametinib in fibroblasts cocultured BRAF-mut melanoma cells. Growth curves of mono-cultured and fibroblasts-cocultured shCtrl 
and SEMA6A-depleted A3 and $\mathrm{H} 2$ 2/59 cells, periodically monitored up to 156 hours (A). The results are presented as mean $+/$ - standard deviation of three independent experimets ( $\left.{ }^{*} p<0.05\right)$. Fold change growth of co-cultured vs mono-cultured shCtrl and SEMA6A-depleted A3 and $\mathrm{H} 2$ cells (B) as reported in (A). Western Blot (WB) analysis of SEMA6A, phosphorylated and total YAP, AKT, P65 and ERK was performed on total cell extracts from shCtrl and SEMA6A-depleted $\mathrm{H} 2$ cells cultured in the absence or presence of fibroblasts for 48 hours. The anti-GAPDH antibody was used to validate equivalent amount of loaded proteins in each lane (C). Growth curves of mono-cultured and co-cultured shCtrl (D) and SEMA6A-depleted $\mathrm{H} 2$ cells $(\mathrm{F})$, untreated or treated with $0,1 \mu \mathrm{M}$ dabrafenib and $0,1 \mu \mathrm{M}$ dabrafenib+5nM trametinib, periodically monitored up to 156 hours. The number of viable mono-cultured and co-cultured shCtrl (E) and SEMA6A-depleted H2 cells (G) 156 hours post-treatment is reported. The results are presented as mean+/- standard deviation of three independent experimets $\left({ }^{*} p<0.05 ; * \star * p<0,0001\right)$. Growth curves of fibroblasts-cocultured shCtrl and SEMA6A-depleted A3 and $\mathrm{H} 2$ cells, untreated or treated as indicated, periodically monitored up to 180 hours. The results are reported as Fold Change number of treated/untreated viable cells $(H)$. Fold Change number of treated/untreated viable co-cultured shCtrl and SEMA6A-depleted $\mathrm{A} 3$ and $\mathrm{H} 2$ cells 180 hours post-treatment is reported (I). The results are presented as mean+/- standard deviation of three independent experimets $\left({ }^{*} p<0.05 ;{ }^{* *} p<0,001\right)$. Western Blot (WB) analysis of SEMA6A, phosphorylated and total YAP, AKT, and ERK was performed on total cell extracts from mono-cultured and fibroblasts-cocultured shCtrl and SEMA6A-depleted H2 cells, untreated and treated as indicated for 48 hours. The anti-HSP70 antibody was used to validate equivalent amount of loaded proteins in each lane $(L)$. 


\section{Figure 6}

A

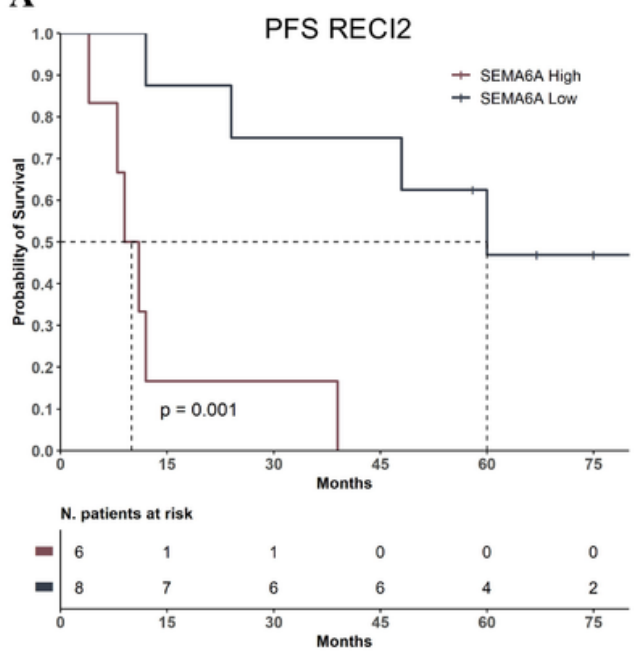

B

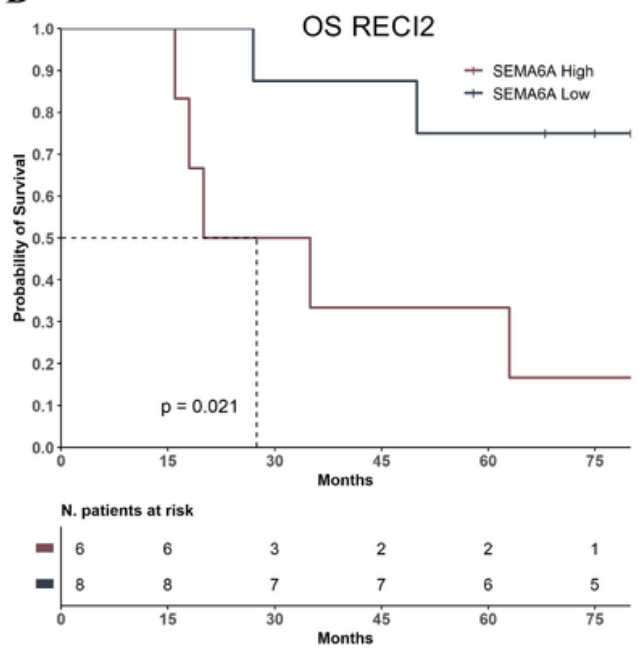

Figure 6

SEMA6A expression predicts low efficacy of dabrafenib+trametinib in BRAF-mut melanoma patients.

Progression-free Survival (PFS) (A) and Overall Survival (OS) (B) in patients with high and low SEMA6A from the cohort RECI2.

\section{Supplementary Files}

This is a list of supplementary files associated with this preprint. Click to download.

- SupplementaryFigureS1.pdf 